Maxwell's fish-eye lens and the mirage of perfect imaging

This article has been downloaded from IOPscience. Please scroll down to see the full text article.

2011 J. Opt. 13024017

(http://iopscience.iop.org/2040-8986/13/2/024017)

View the table of contents for this issue, or go to the journal homepage for more

Download details:

IP Address: 141.211.173.82

The article was downloaded on 06/04/2012 at 16:55

Please note that terms and conditions apply. 


\title{
Maxwell's fish-eye lens and the mirage of perfect imaging
}

\author{
R Merlin \\ Department of Physics, University of Michigan, Ann Arbor, MI 48109-1040, USA \\ Received 3 December 2010, accepted for publication 9 December 2010 \\ Published 21 January 2011 \\ Online at stacks.iop.org/JOpt/13/024017
}

\begin{abstract}
Recent claims that Maxwell's fish-eye is a perfect lens, capable of providing images with deep subwavelength resolution, are examined. We show that the imaging properties of a dispersionless fish-eye are very similar to those of an ideal spherical cavity. Using this correspondence, we prove that the correct solution to Maxwell equations in the fish-eye gives image sizes that are consistent with the standard diffraction limit. Perfect focusing is an optical illusion that results from placing a time-reversed source at the position of the geometrical image which, when combined with the field due to the primary (object) source, mimics the behavior of a perfect drain. Issues of causality are briefly discussed. We also demonstrate that passive outlets are not a good alternative to time-reversed sources for broadband drain-like behavior and that, even if they were, they could not do a better job than conventional optical systems at providing high resolution.
\end{abstract}

Keywords: diffraction limit, subwavelength imaging, metamaterials

(Some figures in this article are in colour only in the electronic version)

Maxwell's fish-eye lens is a sphere of radius $R$ for which the refractive index varies according to

$$
n(r)=\frac{2}{1+(r / R)^{2}},
$$

where $r$ is the distance from the center of the sphere. Inside the lens, ray paths are circles and all rays from an object at $\mathbf{r}_{\mathrm{O}}$ converge at the image point at $\mathbf{r}_{\mathrm{I}}=-\mathbf{r}_{\mathrm{O}} R^{2} /\left|\mathbf{r}_{\mathrm{O}}\right|^{2}$ [1]. The fish-eye lens has recently attracted much attention, motivated by claims that it provides perfect imaging of electromagnetic waves, in an apparent violation of Abbe's diffraction limit [2]. In a series of papers [3-8], Leonhardt et al have argued that the mirrored fish-eye gives unlimited resolution in two [3] and three dimensions [4], and responded to comments by Blaikie [9], Kinsler and Favaro [10], and Merlin [11], who questioned various aspects of their proposal, particularly in regard to the meaning of electromagnetic drains [3-5], causality $[5,11]$, image perfection $[7,8]$, and the assertion that time-reversed sources can be represented by passive outlets [7]. We note that, using transformation optics methods, Benítez et al [12] reached conclusions similar to those of Leonhardt et al for scalar fields in lensing systems other than Maxwell's fish-eye, whereas Guenneau et al failed to observe deep subwavelength resolution in both Maxwell's fish-eye and the Eaton lens [13].
Here, we compare Maxwell's fish-eye to the mathematically simpler problem of a spherical mirror, and find strong similarities in their imaging behavior. We used this analogy to show that the perfect focusing claimed by Leonhardt et al $[3,4]$ is not an intrinsic property of the fish-eye lens, but merely the result of having placed an additional source at the image position. In this regard, their proposal is reminiscent of schemes relying on time-reversed sources to attain subwavelength focusing $[14,15]$. The extra source leads to a resulting field pattern that imitates the behavior of a drain. Leonhardt et al assert that drains are necessary to achieve a stationary state and that solutions without them violate causality [4]. In contrast, we find that the problem of a single dipole in an ideal spherical cavity does exhibit causal stationary states without the need for drains, and that the images obtained in that situation obey Abbe's constraint. Disproving the belief that mirrors are not good analogs of Maxwell's lens [7, 8] our analysis shows that, as for the dispersionless fish-eye, electromagnetic pulses do not experience shape distortion as they propagate in large spherical cavities. Finally, we show that passive outlets can imitate drains, but only at a single frequency (this is consistent with a recent study by Kinsler [16]) and under conditions that require fine tuning of parameters. 


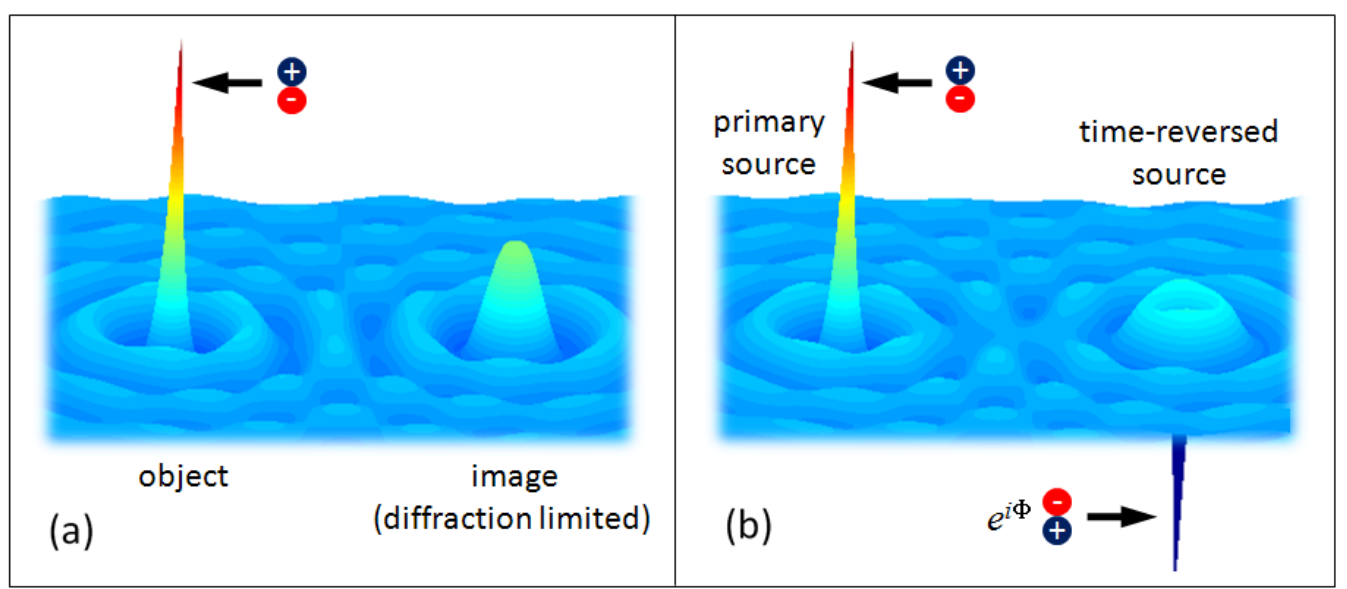

Figure 1. A cartoon view of imaging in Maxwell's fish-eye and ellipsoidal cavities. (a) A single dipole source gives diffraction-limited focusing. (b) The result of adding a second, time-reversed electric dipole at the position of the image is a field pattern that emulates that of a drain.

The problem of a point electric-dipole source at the center of a spherical cavity can be solved exactly $[11,17]$. We assume that the dipole is driven with a current whose time dependence is known a priori to be of the form $\exp (-\mathrm{i} \omega t)$, and which cannot be modified by the radiation fields. The sphere, of radius $R$, is covered on the inside with a perfectly reflecting material. Ray optics dictates that the image is also at the center of the sphere. For a dipole oriented along the $z$-axis, the harmonic solutions for the Hertz vector are of the form $\boldsymbol{\Pi}=\Pi_{\omega} \mathrm{e}^{-\mathrm{i} \omega t} \mathbf{e}_{z}$ where

$$
\Pi_{\omega}=k p_{0}\left(\frac{\mathrm{e}^{\mathrm{i} k r}}{k r}+\mathrm{i} A \frac{\sin k r}{k r}\right) ;
$$

$k=\omega / c$ ( $c$ is the speed of light). This gives $\mathbf{H}=H_{\varphi} \mathrm{e}^{-\mathrm{i} \omega t} \mathbf{e}_{\varphi}$ and $\mathbf{E}=\left(E_{r} \mathbf{e}_{r}+E_{\theta} \mathbf{e}_{\theta}\right) \mathrm{e}^{-\mathrm{i} \omega t}$ with

$$
\begin{gathered}
H_{\varphi}=H_{d}+A k^{3} p_{0} j_{1}(k r) \sin \theta \quad E_{\theta}=-\frac{\mathrm{i}}{k r} \frac{\partial}{\partial r}\left(r H_{\varphi}\right) \\
E_{r}=\mathrm{i} \frac{2 H_{\varphi}}{k r \tan \theta} .
\end{gathered}
$$

Here, $\mathbf{H}$ and $\mathbf{E}$ are the magnetic and electric field, $j_{1}$ and $h_{1}^{(1)}$ are the first-order spherical Bessel and first-kind Hankel functions, and $H_{d}=k^{3} p_{0} h_{1}^{(1)}(k r) \sin \theta$ is the contribution of the dipole alone, which gives outgoing waves $\propto \mathrm{e}^{\mathrm{i} \omega(r / c-t)} / k r$ for $k r \gg 1$. From the boundary condition, requiring that $E_{\theta}=0$ at $r=R$, we get

$$
A=-\frac{h_{1}^{(1)}(k R)+k R h_{1}^{(1)^{\prime}}(k R)}{j_{1}(k R)+k R j_{1}^{\prime}(k R)}
$$

where the prime indicates the derivative with respect to $k R$. Note that the boundary condition for $k R \gg 1$ is $\Pi_{\omega}(R)=0$.

In equation (1), the non-singular solution to the homogeneous equation, $\sin (k r) / k r$, represents the field due to the induced currents at the inner surface of the sphere. It is apparent that this term gives a diffraction-limited image at $r=0$. The corresponding expression for scalar waves in the fish-eye lens $[7,8]$ is

$$
D_{\omega} \approx \frac{\sin (2 \omega \Delta)}{16 \pi^{2} \Delta \sin (\pi \omega)},
$$

which is also consistent with Abbe's standard diffraction limit; $\Delta=\left|\mathbf{r}-\mathbf{r}_{\mathrm{I}}\right| \ell /\left|r_{\mathrm{I}}^{2}+\ell^{2}\right|$. Leonhardt et al assert generally that 'to maintain a stationary regime, we must supplement the source by a drain' [4]. However, given that the fields in equations (1) and (2) are drain-free, it is clear that this statement is incorrect. Thus, there is no need to include a drain to achieve a steady state.

Let us now add a second dipole $p_{0}^{\prime}=\alpha p_{0}$, also oriented along the $z$-axis, at the center of the sphere, and choose $\alpha$ so that its associated field exactly cancels the outgoing wave due to the induced currents (the two dipoles coincide here because both object and image are at $\mathbf{r}=0$; in the fish-eye or an ellipsoidal cavity, the conjugate foci and, thus, the dipoles occupy different positions). That is, we require that

$$
\alpha=-\frac{A}{2+A}=\frac{h_{1}^{(1)}(k R)+k R h_{1}^{(1)^{\prime}}(k R)}{h_{1}^{(2)}(k R)+k R h_{1}^{(2)^{\prime}}(k R)}=-\mathrm{e}^{\mathrm{i} \Phi}
$$

so that

$$
\Pi_{\omega}=k p_{0}\left(\frac{\mathrm{e}^{\mathrm{i} k r}}{k r}-\mathrm{e}^{\mathrm{i} \Phi} \frac{\mathrm{e}^{-\mathrm{i} k r}}{k r}\right) .
$$

This expression, emphasizing the drain-like look of the resulting field, is analogous to what Leonhardt et al found for the mirror-coated fish-eye [3,4]. It is easy to see that what Leonhardt et al refer to as 'subwavelength imaging' is nothing more than the singularity introduced by the extra source of radiation. Similar to the results for the fish-eye, the phase shift is $\Phi \approx 2 k R+\pi$ for $k R \gg 1$. The cartoons in figure 1 provide a comparison between the cases represented by equations (1) and (3). We emphasize that both expressions are physical, causal solutions to different problems (involving, respectively, one and two sources), none of which contains a drain.

Consider now imaging in the time domain. For wavelengths that are significantly shorter than the radius of the 

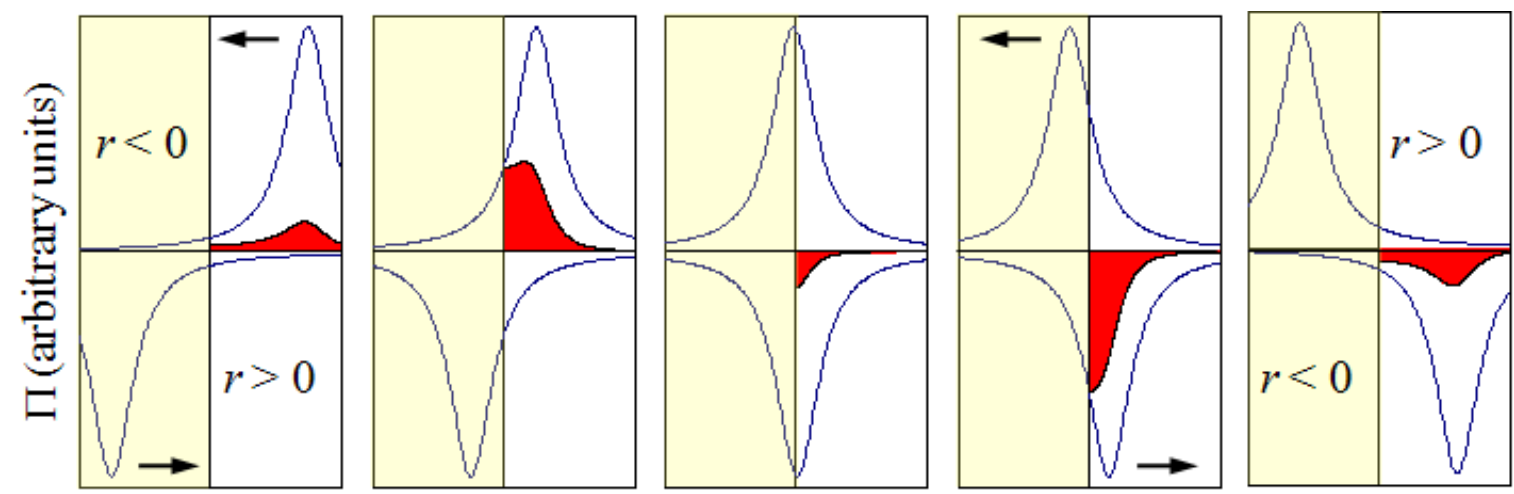

Figure 2. Time domain focusing. The solid black curves delimiting the red shaded areas are $\Pi$ versus $r$ (arbitrary units) at $t \sim 2 m R / c$; see equation (4). Time runs from left to right. Waves for $r>0(r<0)$ are real (virtual). Interference between the incoming and outgoing waves (blue curves) gives the diffraction-limited image at $r=0$, with $\Pi=0$ at $t=2 \mathrm{mR} / \mathrm{c}$. Drain-like behavior results from adding a time-reversed source at the image position that exactly cancels the outgoing wave. (Color online.)

sphere, we can approximate $A \approx \mathrm{ie}^{\mathrm{i} k R} / \sin k R$. Thus

$$
\begin{aligned}
& \Pi(r, t)=\frac{R}{2 \pi c} \int_{-\infty}^{+\infty} \Pi_{\omega} \mathrm{e}^{-\mathrm{i} \omega t} \mathrm{~d} \omega \\
& \quad \approx \frac{p_{0} R}{2 \pi r} \int_{-\infty}^{+\infty}\left(\mathrm{e}^{\mathrm{i} k r}-\frac{\mathrm{e}^{\mathrm{i} k r}-\mathrm{e}^{-\mathrm{i} k r}}{1-\mathrm{e}^{-2 \mathrm{i} k R}}\right) \mathrm{e}^{-\mathrm{i} \omega t} \mathrm{~d} k .
\end{aligned}
$$

Applying Cauchy's theorem, we get

$$
\begin{aligned}
& \Pi(r, t)=\frac{p_{0} R}{r}\left[\delta(c t-r)-\frac{\Theta(t)}{2 R}\right. \\
& \left.\times \sum_{m=-\infty}^{\infty}\left(\mathrm{e}^{-\mathrm{i} m \pi(c t-r) / R}-\mathrm{e}^{-\mathrm{i} m \pi(c t+r) / R}\right)\right] \\
& =\frac{p_{0} R}{r}\left\{\delta(c t-r)-\Theta(t) \sum_{m=-\infty}^{\infty}[\delta(c t-r-2 m R)\right. \\
& -\delta(c t+r-2 m R)]\}
\end{aligned}
$$

where $\Theta(t)$ is the Heaviside (step) function. The above expression indicates that a pulse emitted by the source periodically reflects off the inner mirrored surface and converges at the center of the sphere without distortion. The $r$-dependence of $\Pi$ is shown in figure 2 at times when the pulse is close to $r=0$. The diffraction-limited field at the image site can be thought of as resulting from the interference between an incoming and an outgoing wave. The introduction of a second, time-reversed source, which interferes destructively with the outgoing wave, creates the mirage of a drain. If one ignores the wavelength dependence of the refractive index, a nearly identical result can be obtained for the fish-eye $[7,8]$

$$
\begin{aligned}
D= & \int_{-\infty}^{+\infty} D_{\omega}(\mathbf{r}) \mathrm{e}^{-\mathrm{i} \omega t} \mathrm{~d} \omega=\frac{\Theta(t)}{8 \pi}\left(\Delta+\frac{1}{\Delta}\right) \\
& \times \sum_{m=-\infty}^{+\infty}\left[\delta\left(c t / \ell-2 \tan ^{-1} \Delta-2 m \pi\right)\right. \\
& \left.-\delta\left(c t / \ell+2 \tan ^{-1} \Delta-2 m \pi\right)\right] .
\end{aligned}
$$

It follows that the statement by Leonhardt et al that 'Maxwell's fish-eye has imaging properties different from mirrors' [7] is inaccurate. We notice that, even though subwavelength resolution and chromatic aberration (pulse dispersion) are unrelated problems, Leonhardt et al treat the absence of pulse broadening as a requirement for perfect imaging [7]. We also note that their statement that 'in imperfect imaging, the drain would be in conflict with causality' [8] is mistaken since equation (3) applies for all values of $k R$, including the range $k R<1$ for which the pulses experience significant chromatic distortion.

Finally, we discuss the possibility that time-reversed sources may be replaced by passive outlets. Leonhardt et al [6] claim that 'Maxwell's fish-eye [...] makes a perfect lens for electromagnetic waves but only when such waves are detected by perfect point detectors'. As we have seen, the spherical mirror has properties similar to those of the fish-eye lens. Can a passive detector at the origin lead to behavior that imitates a drain in the spherical mirror? As recent work has shown for the general case [16], the answer is yes, but only at a single predetermined frequency. Here, we give an alternative argument. Detectors of dimensions $\sim \ell$ that are small compared with the wavelength $\lambda$ behave as particles in a uniform field [18]. Let $p_{\mathrm{D}}$ be the electric dipole acquired by the detector and $\gamma_{\mathrm{D}}$ its electrical polarizability (we ignore magnetic effects). A calculation of the field at the origin, due to the induced currents at the mirror, gives $\mathbf{E}(0)=E(0) \mathbf{e}_{z}$ where $E(0)=(2 \mathrm{i} / 3) A k^{3}\left(p_{0}+p_{\mathrm{D}}\right)$. A perfect drain-like pattern requires that $p_{\mathrm{D}}=\gamma_{\mathrm{D}} E(0)=-p_{0} \mathrm{e}^{\mathrm{i} \Phi}$ which gives

$$
\gamma_{\mathrm{D}}=3 \mathrm{i} / 4 k^{3} \text {. }
$$

Since the polarizability is proportional to the volume of the detector, it is clear that this condition together with $\ell \ll$ $\lambda$ can only be met in the vicinity of a high- $Q$ resonance and at a single frequency. As an example, if we model the outlet as a sphere of radius $r_{\mathrm{D}}$ and permittivity $\varepsilon_{\mathrm{D}}$, the polarizability is $\gamma_{\mathrm{D}}=(\varepsilon-1) r_{\mathrm{D}}^{3} /(\varepsilon+2)$ [18], and the relevant resonance is that of the so-called surface plasmon. Therefore, the condition set by equation (5) reads $\varepsilon_{\mathrm{D}} \approx$ $-2+4 \mathrm{i} k^{3} r_{\mathrm{D}}^{3}$. Similar considerations apply to the microwave experiments reported by Ma et al [19] in which the outlets were absorbers identical to the source and impedance matched to the cables, which thus behaved as sources in reverse. It is important to note that, even if passive elements could be 
used for broadband operation, their associated drains would not provide subwavelength resolution. The reason is that the spatial dependence of the amount of radiation emitted or absorbed by the device would still be limited by diffraction effects and, as such, by the same signal-to-noise problems that arise when trying to resolve objects whose separation is smaller than that allowed by Abbe's formula.

\section{Acknowledgment}

Work supported by the Air Force Office of Scientific Research under contract FA 9550-06-01-0279 through the Multidisciplinary University Research Initiative Program.

\section{References}

[1] Maxwell C M 1952 The Scientific Papers of James Clerk Maxwell vol 1, ed W D Niven (New York: Dover) p 76

The fish-eye lens is mentioned for the first time in a problem and its solution, both attributed to Maxwell:

1853 Cambridge Dublin Math. J. 8188

1853 Cambridge Dublin Math. J. 99
[2] Abbe E 1873 Arch. Mikrosk. Anat. 9413

[3] Leonhardt U 2009 New J. Phys. 11093040

[4] Leonhardt U and Philbin T G 2010 Phys. Rev. A 81 011804(R)

[5] Leonhardt U 2010 New J. Phys. 12058002

[6] Leonhardt U 2010 arXiv: 1010.4161

[7] Leonhardt U and Philbin T G 2010 Phys. Rev. A 82057802

[8] Leonhardt U and Sahebdivan S 2010 J. Opt. 13024016 (this issue) (arXiv:1007.0078)

[9] Blaikie R J 2010 New J. Phys. 12058001

[10] Kinsler P and Favaro A 2010 New J. Phys. submitted (arXiv:1010.2346)

[11] Merlin R 2010 Phys. Rev. A 82057801

[12] Benítez P, Miñano J C and González J C 2010 Opt. Express 187650

[13] Guenneau S, Diatta A and McPhedran R C 2010 J. Mod. Opt. 57511

[14] Lerosey G, de Rosny J and Fink M 2007 Science 3151120

[15] de Rosny J and Fink M 2002 Phys. Rev. Lett. 89124301

[16] Kinsler P 2010 Phys. Rev. A 82055804

[17] Butler C M and van Bladel J 1964 IEEE Trans. Antennas Propag. 12110

[18] Landau L D and Lifshitz E M 1960 Electrodynamics of Continuous Media (Oxford: Pergamon) p 319

[19] Ma Y G, Ong C K, Sahebdivan S, Tyc T and Leonhardt U 2010 arXiv: 1007.2530 